\title{
Placental pathology in systemic lupus erythematosus: a case series
}

Priyani AAH, de Silva MVC

\section{Introduction}

Priyani AAH

Systemic Lupus Erythematosus (SLE) is a multisystem autoimmune disease that can compromise pregnancy, its outcome and is the connective tissue disease that is most frequently associated with pregnancy (1). we report the findings in a series of patients The presence of lupus anticoagulant, found in up to a third of patients, has been reported to be a positive predictor of increased foetal loss after 20 weeks' gestation in women with lupus nephritis (2). Pathological changes in the placenta have been detected as early as the first trimester of pregnancy (3). There are no reports of the placental pathological findings of SLE patients in Sri Lanka, and diagnosed clinically with SLE.

\section{Case reports}

The placentae of six patients with SLE were examined grossly and tissue sections were taken from abnormal areas, representative normal placental parenchyma, umbilical cord and membranes. The microscopic findings were assessed.

\section{Pathological findings}

Placentae of case number 1, 2, 3 and 4 were small and showed distal villous hypoplasia characterized by small, sparse villi with large syncytial knots over the surface. (Figure 1). The placentas of case number 2 and 3 showed decidual vasculopathy characterized by acute atherosis and thrombosis with luminal occlusion of decidual blood vessels. The vessels with acute atherosis showed fibrinoid necrosis of the vessel wall and deposition of foamy histiocytes in the intima (Figure 2).

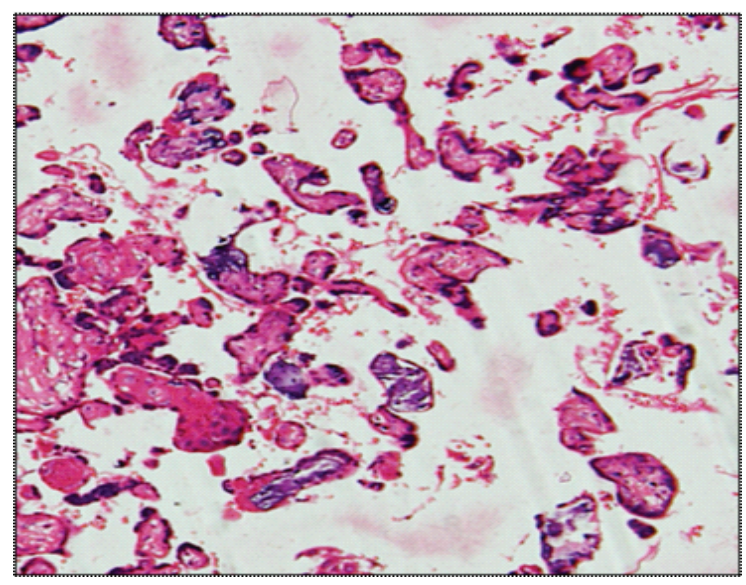

Fig 1. Distal villous hypoplasia (H\& EX10)

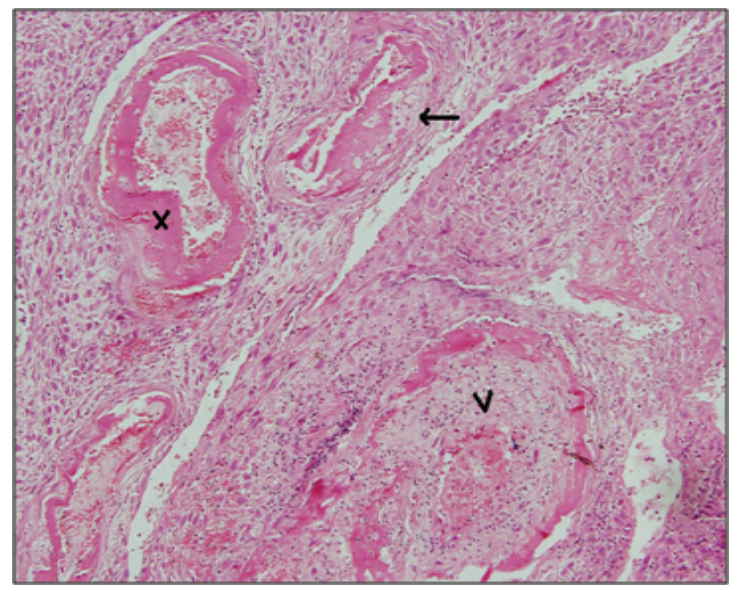

Fig 2. Decidual arteriopathy in case 2 (H\&EX10) X-Fibrinoid necrosis of the vessel wall $\mathrm{V}$ - Thrombosis with luminal occlusion (Arrow - Acute atherosis of the intima)

Department of Pathology, Faculty of Medicine, University of Colombo, Sri Lanka.

Correspondance: Priyani AAH, Department of Pathology, Faculty of Medicine, University of Colombo, Sri Lanka.e-mail: priyaniaah@yahoo.com 
Both placentas showed multiple infarctions and areas with increased inter villous and peri villous fibrin deposition affecting a significant proportion of the placental volume. Placentas of case showed evidence of ascending infection as a second pathology. Case number 6 had a good volume placenta with no abnormalities.

\section{Discussion}

The pathological findings in this case series is similar to larger series of studies $(4,5)$. Both hypertension and vasculopathies related to lupus anticoagulant will result in maternal vascular supply abnormalities in the placenta. Early abnormalities in placental development is in the process of placental implantation where trophoblastic infiltration of the endometrium and myometrium and remodelling of the uterine spiral arteries occurs. Superficial implantation will lead to decidual arteriopathy, initially manifesting as mural hypertrophy of the muscular walls and persistence of muscularized arteries in the basal plate and later as fibrinoid necrosis of the vessel wall with collection of foamy histiocytes in the intima (acute atherosis). Severe reduction in uterine blood flow can lead to undergrowth of the placental villous tree manifesting as distal villous hypoplasia, increased syncytial knots on distal villi, deposition of intervillous fibrin on proximal and distal villi, villous agglutination and decreased placenta 1 number 4 and 5 showed similar lesions involving $<10 \%$ of the placental volume. An inter villous thrombus and bleeding into desidua were seen in case number 4 . Case number 1 weight. Total occlusion of one or more maternal arteries leads to infarction of a portion of the villous tree $(6,7)$. Studies have shown that placental pathological findings correlate well with the clinical outcome (8). Pathological examination of the placenta is often neglected. Histological examination gives valuable information to identify the underlying pathology of cases with adverse fetal outcome.

\section{Acknowledgements}

The authors acknowledge the technical officers in the Department of Pathology, for the technical assistance provided.

\section{References}

1. De Castro Surita FG, Parpinelli MA, Yonehara E, Kurupa F, Cecatti JG. Systemic lupus erythematosus and pregnancy: clinical evolution, maternal and perinatal outcomes and placental findings. Sao Paulo Medical Journal 2007; 125(2): 91-5.

2. Simpson L L. Maternal Medical Disease: Risk of Antepartum Fetal Death Seminars in Perinatology February 2002; 26(1): 42-50.

3. Nayar R, Lage JM. Placental changes in a first trimester missed abortion in maternal systemic lupus erythaematosus with antiphospholipid syndrome; a case report and review of literature Human Pathology February 1996; 27(2): 201-206.

4. Ogishima D, Matsumoto T, Nakamura Y, Yoshida $\mathrm{K}$, Kuwabara Y Placental pathology in systemic lupus erythematosus with antiphospholipid antibodies. Pathology International March 2000; 50(3): 224-9.

5. Magid MS, Kaplan C, Sammaritano LR, Peterson M, Druzin ML, Lockshin MD. Placental pathology in systemic lupus erythematosus: a prospective 
study. American Journal of Obstetrics and Gynecology July 1998; 179(1): 226-34.

6. Redline R W. Placental Pathology: A Systematic Approach with Clinical Correlations Placenta 2008; 29(Supplement A), Trophoblast Research; (22): 86-91.

7. Yee Khong T. The placenta in stillbirth Current Diagnostic Pathology 2006; 12: 161-172.

8. Stonea S, Pijnenborgc R, Vercruyssec L, Postond R, Khamashtab M A, Huntb, B J et al. The Placental Bed in Pregnancies Complicated by Primary Antiphospholipid Syndrome Placenta 2006; 27 : 457-467. 